\title{
EATING LATE AND LESS FREQUENTLY HAS A NEGATIVE IMPACT ON THE DIET QUALITY OF BREAST CANCER WOMEN UNDERGOING ENDOCRINE THERAPY
}

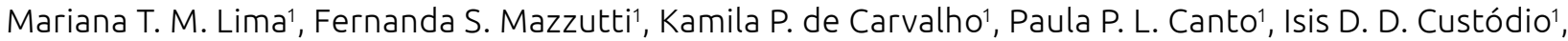
Cibele A. Crispim¹', Carlos E. Paiva', Yara C. de P. Maia ${ }^{1}$

${ }^{1}$ Universidade Federal de Uberlândia - Uberlândia (MG), Brazil.

This cross-sectional study aimed to verify the effect of meal timing and eating frequency on quantitative and qualitative food consumption, as well as the physical and biochemical measurements of breast cancer women undergoing endocrine therapy with tamoxifen. Quantitative dietary assessments of 84 women were performed by applying three nonconsecutive $24 \mathrm{~h}$ dietary recalls. The Brazilian Healthy Eating Index Revised (BHEI-R) was used for the qualitative analysis of diet. Participants were dichotomized in Early and Late eaters for the main meals using the median values of hours as the cut-off point. The eating frequency was defined as the number of times per day of food or beverage consumption with at least $5 \mathrm{kcal}$ and a minimum difference of 60 minutes between each eating episode. Body composition and biochemical measurements were investigated. The percentage values of the body fat were significantly higher among women with consumption $\leq 4$ eating episodes when compared to those that consumed $>4$ per day. Furthermore, the Late dinner time women had significantly lower plasma levels of high density lipoprotein (HDL-C) compared to those of Early dinner time. Significant effects of eating frequency were observed for quantitative intake of energy and macronutrients, however no effects of main meal timing. Regarding the quality of diet, positive associations were observed for Early women: for breakfast time to Whole Grains; Milk and Dairy Products - for dinner time: to Calories from Solid Fats, Alcohol and Added Sugars and the Total BHEI-R score. The consumption $\geq 4$ eating episodes was positively associated with consumption of Total Fruits, Whole Fruits, Meat, Eggs, and Beans and the Total BHEI-R score compared with women who consumed $<4$ per day. Therefore, late eaters and less eating frequency presented dietary patterns of lower quality, worse physical, and biochemical measurements, that could have an adverse impact in the health of women in endocrine treatment. 